\title{
Discrete Quantum Transitions, Duality: Emergence of Physical Structures and Occurrence of Observed Formations (Hidden Properties of Mathematical Physics Equations)
}

\author{
Ludmila Petrova \\ Department of Computational Mathematics and Cybernetics, Moscow State University, Moscow, Russia \\ Email: ptr@cs.msu.su
}

How to cite this paper: Petrova, L. (2020) Discrete Quantum Transitions, Duality: Emergence of Physical Structures and Occurrence of Observed Formations (Hidden Properties of Mathematical Physics Equations). Journal of Applied Mathematics and Physics, 8, 1911-1929.

https://doi.org/10.4236/jamp.2020.89144

Received: July 16, 2020

Accepted: September 20, 2020

Published: September 23, 2020

Copyright $\odot 2020$ by author(s) and Scientific Research Publishing Inc. This work is licensed under the Creative Commons Attribution International License (CC BY 4.0). http://creativecommons.org/licenses/by/4.0/

\begin{abstract}
With the help of skew-symmetric differential forms, the hidden properties of the mathematical physics equations that describe discrete quantum transitions and emergence the physical structures are investigated. It is shown that the mathematical physics equations possess a unique property. They can describe discrete quantum transitions, emergence of physical structures and occurrence observed formations. However, such a property possesses only equations on which no additional conditions, namely, the conditions of integrability, are imposed. The intergrability conditions are realized from the equations themselves. Just under realization of integrability conditions double solutions to the mathematical physics equations, which describe discrete transitions and so on, are obtained. The peculiarity consists in the fact that the integrability conditions do not directly follow from the mathematical physics equations; they are realized under the description of evolutionary process. The hidden properties of differential equations were discovered when studying the integrability of differential equations of mathematical physics that depends on the consistence between the derivatives in differential equations along different directions and on the consistence of equations in the set of equations. The results of this work were obtained with the help of skew-symmetric differential forms that possess a nontraditional mathematical apparatus such as nonidentical relations, degenerate transformations and the transition from nonintegrable manifolds to integrable structures. Such results show that mathematical physics equations can describe quantum processes.
\end{abstract}

\section{Keywords}

Integrability Conditions of Differential Equations, Double Solutions, Realization 
of Integrable Structures, Discrete Transitions, Emergence of Various Structures and Observed Formations

\section{Introduction}

In present paper we study partial differential equations and sets of mathematical physics equations that describe any processes and on what no additional conditions are imposed.

When studying the integrability of differential equation in partial derivatives one obtains that on an initial coordinate space such equations are not integrable ones. The derivatives of such equations do not made up a differential. The solutions are not functions depending only on variables. However, under the presence of any degrees of freedom it can be realized some additional conditions (conditions of integrability) at which integrable structures are realized. The solution on integrable structures becomes a discrete function. The transition from initial coordinate space to integrable structure and the realization of discrete function describes discrete transitions, quantum jumps, and the advent of any structures. To this case it corresponds a degenerate transformation, i.e. a transformation that does not conserve a differential and which can be describe only by skew-symmetric differential forms.

Here it should clue attention to the fact that the emergence of various jumps and structures proceeds under realization of additional conditions, namely, the conditions of integrability that, in the present case, depend on the consistence of derivatives along different directions.

The set of differential equations also possesses such properties if no additional conditions are imposed on that. The emergence of various jumps and structures also take place under the realization of additional conditions, namely, the conditions of integrability. However, in the present case the integrability depends on the consistence of equations that made up the set of equations. When studying the consistence of equation contained in the set of equations one obtains an evolutionary relation in terms of skew-symmetric differential forms that describe an emergence of various structures.

The results of present paper were obtained with the help of skew-symmetric differential forms [1] [2] [3]. In this case, in addition to closed exterior skewsymmetric differential forms [1], which possess the invariant properties, it has been used the skew-symmetric differential forms, which basis are non-integrable manifolds (as opposed to exterior skew-symmetric differential forms whose basis are integrable structures). These skew-symmetric forms, unlike closed exterior forms, are evolutionary ones. Such evolutionary forms (which contain an unconventional mathematical apparatus that includes such basic concepts as degenerate transformations and nonidentical relations) can describe evolutionary processes and generate closed exterior forms corresponding to invariant structures. 
Some properties of skew-symmetric forms are given in [4] and in Appendix 1.

\section{Study of Integrability of Differential Equation in Partial Derivatives, Double Solutions, Emergence of Physical Structures}

As it was already noted, the integrability of differential equation in partial derivatives depends on the consistence between derivatives along different directions.

\subsection{Investigation of Consistence between Derivatives of Differential Equation: Solutions on the Original Coordinate Space}

The basic idea of the qualitative investigation of differential equations can be clarified by the example of the first-order partial differential equation [5].

Let

$$
F\left(x^{i}, u, p_{i}\right)=0, \quad p_{i}=\partial u / \partial x^{i}
$$

be the partial differential equation of the first order.

In order to Equation (1) be integrable, the derivatives of that equation must be consistent, that is, they must made up a differential.

Let us consider the functional relation

$$
\mathrm{d} u=\theta
$$

where $\theta=p_{i} \mathrm{~d} x^{i} \quad$ (the summation over repeated indices is implied). Here $\theta=p_{i} \mathrm{~d} x^{i}$ is a skew-symmetric differential form of the first degree [3] (see Appendix 1).

If relation (2) is identical one, that is, the differential form $\theta=p_{i} \mathrm{~d} x^{i}$ is a differential (as the left-hand side of relation (2)), this relation can be integrated. This means the solution $u$ of the differential equation will be a function, that is, it will depend only on variables $x^{i}$.

The specific feature of functional relation (2) is that, in the general case, when differential Equation (1) describes any physical processes, this relation turns out to be nonidentical one.

In the general case, from equation (1) it does not follow (explicitly) that the derivatives $p_{i}=\partial u / \partial x^{i}$, which obey to the equation (and given boundary or initial conditions of the problem), made up a differential. That is, from Equation (1) it does not follow that the differential form $\theta=p_{i} \mathrm{~d} x^{i}$ is a differential (like the left-hand side in relation (2)).

For differential form $\theta$ be a differential, it is necessary that the mixed derivatives of the function $u$ must be permutable. That is, the commutator $K_{i j}=\partial p_{j} / \partial x^{i}-\partial p_{i} / \partial x^{j}$ of the differential form $\theta$ must vanish.

For equations descriptive of any processes (without any supplementary conditions), the commutator $K_{i j}$ of the differential form $\theta$ is not equal to zero. The form $\theta=p_{i} \mathrm{~d} x^{i}$ turns out to be unclosed and is not a differential like the left-hand 
side of relation (2). Functional relation (2) appears to be nonidentical one: the left-hand side of this relation is a differential whereas the right-hand side is not a differential.

The nonidentity of the functional relation (2) points to the fact, that without additional conditions the derivatives of the initial equation do not constitute a differential. This means that the solution $u$ to differential equation on the original coordinate space will not be a function of $x^{i}$. It will depend on the commutator of the form $\theta$, that is, the solution $u$ will depend not only on the variables, the derivatives of solution do not made up a differential.

Under what conditions can the solution to differential equation become a function?

\subsection{Realization of Differential Equation Integrability, Solutions to Differential Equations on Integrable Structures}

To obtain the solution that is a function (i.e. the derivatives of this solution made up a differential), it is necessary that the closure condition of the form $\theta=p_{i} \mathrm{~d} x^{i}$ and the closure condition of its dual form be satisfied (in the present case the functional $F$ plays a role of the form dual to $\theta$ ). That is, the differentials of the form $\theta$ and its dual form have to be zero;

$$
\left\{\begin{array}{l}
\mathrm{d} F\left(x^{i}, u, p_{i}\right)=0 \\
\mathrm{~d}\left(p_{i} \mathrm{~d} x^{i}\right)=0
\end{array}\right.
$$

(Here it should be noted that the form $\theta$ can become only inexact closed exterior skew-symmetric form, i.e. a form closed only on a certain structure described by a closed dual form).

If we expand the differentials, we get a set of homogeneous equations with respect to $\mathrm{d} x^{i}$ and $\mathrm{d} p_{i}$ :

$$
\left\{\begin{array}{l}
\left(\frac{\partial F}{\partial x^{i}}+\frac{\partial F}{\partial u} p_{i}\right) \mathrm{d} x^{i}+\frac{\partial F}{\partial p_{i}} \mathrm{~d} p_{i}=0 \\
\mathrm{~d} p_{i} \mathrm{~d} x^{i}-\mathrm{d} x^{i} \mathrm{~d} p_{i}=0
\end{array}\right.
$$

The solvability condition for this set, namely, vanishing the determinant composed of the coefficients at $\mathrm{d} x^{i}$ and $\mathrm{d} p_{i}$, have the form:

$$
\frac{\mathrm{d} x^{i}}{\partial F / \partial p_{i}}=\frac{-\mathrm{d} p_{i}}{\partial F / \partial x^{i}+p_{i} \partial F / \partial u}
$$

These conditions determine an integrable structure, namely, a pseudostructure (in its metric properties), on which the form $\theta=p_{i} \mathrm{~d} x^{i}$ turns out to be closed, i.e. it becomes a differential, so that from relation (2) the identical relation is deduced. If conditions (5), which may be called "integrability conditions", are satisfied, the derivatives constitute a differential $\delta u=p_{i} \mathrm{~d} x^{i}=\mathrm{d} u$ (on the pseudostructure), and the solution becomes a function. The function $u\left(x^{i}\right)$, where $x^{i}$ are coordinates of the point on pseudostructure, is a discrete function, i.e. a so-called generalized solutions. 
Since functions that are the generalized solutions are defined only on integrable structures, they have discontinuities in their derivatives in directions that are transverse to integrable structures [6]. The order of the derivatives with discontinuities is equal to the degree of the exterior form. If a form of zero degree be involved in the functional relation, the function itself, being a generalized solution, will have discontinuities.

[If we find the characteristics of Equation (1), it appears that conditions (5) are the equations for the characteristics. That is, the characteristics are examples of integrable structures (the pseudostructures) on which the derivatives of the differential equation consist of closed forms and the solutions prove to be the functions (generalized solutions)].

\subsection{Discrete Transitions, Emergence of Physical Structures}

Closed dual form (the pseudostructure, i.e. the integrable structure) and closed inexact exterior form (the conservative quantity) which are realized under degenerate transformations, made up a differential-geometric structure, i.e. a pseudostructure with conservative quantity.

Such differential-geometrical structure describes physical structure, i.e. the conservative invariant objects obeying conservation laws.

The transition from the solution on original coordinate space to the solution on integrable structure describes a discrete transition and emergence of a physical structures. This happens discretely, that is, by quanta.

The characteristics, the singular points, the envelopes of characteristics, and other structures with conserved quantities are examples of such physical structure.

In Section 4 it will be shown that the emergence of physical structures is accompanied with the appearance of observed formations, such as fluctuations, waves, turbulent pulsations, etc. The physical meaning of such processes will be revealed.

Here it should call attention to the nontraditional mathematical apparatus, such as nonidentical relations, degenerate transformations and the transition from nonintegrable manifolds to integrable structures. These can be described only with the help of skew-symmetric differential forms [3].

Thus one can see that the conditions of integrability of differential equations on which no additional conditions are imposed can be directly realized from differential equations (this is possible when there are any degrees of freedom) and this describes discrete transitions, breaks and the emergence of physical structures.

It should be noted that the integrability condition is realized when the determinant becomes zero (this corresponds to degenerate transformation, i.e. to the transformation that does not conserve a differential), and under the transition from tangent manifold made up of derivatives of initial Equation (1) to cotangent manifold. This may be seen by the example of the equation resolved with respect to time. 


\section{The Investigation of Integrability of Differential Equation Resolved with Respect to Time}

The investigation of integrability of differential equations can be clarified by the example of the first-order partial differential equation (see (1)) that does not explicitly depend on $u$ and is solved with respect to some variable, for example $t$, that is, it has the form [5]

$$
\frac{\partial u}{\partial t}+E\left(t, x^{j}, p_{j}\right)=0, \quad p_{j}=\frac{\partial u}{\partial x^{j}}
$$

Then the integrability conditions (5) take the form (in this case $\partial F / \partial p_{1}=1$ )

$$
\frac{\mathrm{d} x^{j}}{\mathrm{~d} t}=\frac{\partial E}{\partial p_{j}}, \quad \frac{\mathrm{d} p_{j}}{\mathrm{~d} t}=-\frac{\partial E}{\partial x^{j}}
$$

The conditions (7) are known as the canonical relations. The Equation (6) provided with the supplementary conditions, namely, the canonical relations (7), is called the Hamilton-Jacobi equation. The derivatives of this equation made up the differential: $\delta u=(\partial u / \partial t) \mathrm{d} t+p_{j} \mathrm{~d} x^{j}=-E \mathrm{~d} t+p_{j} \mathrm{~d} x^{j}=\mathrm{d} u$.

To this type there belongs the equation of field theory

$$
\frac{\partial s}{\partial t}+H\left(t, q_{j}, \frac{\partial s}{\partial q_{j}}\right)=0, \quad \frac{\partial s}{\partial q_{j}}=p_{j}
$$

where $s$ is the field function for the action functional $S=\int L \mathrm{~d} t$. Here $L$ is the Lagrange function, $H$ is the Hamilton function: $H\left(t, q_{j}, p_{j}\right)=p_{j} \dot{q}_{j}-L$, $p_{j}=\partial L / \partial \dot{q}_{j}$.

To the Equation (8) there correspond the closed form $\mathrm{d} s=H \mathrm{~d} t+p_{j} \mathrm{~d} q_{j}$ (the Poincare invariant) [In the quantum theory an analog to the Equation (8) is the Schrödinger equation].

Here the degenerate transformation is a transition from the Lagrange function to the Hamilton function. An equation for the Lagrange function, which is the Euler variational equation, has been obtained from the condition $\delta S=0$, where $S$ is the action functional. In the real case, when forces are nonpotential or connectednesses are nonholonomorphic, the quantity $\delta S$ is not a closed form, that is, $\mathrm{d} \delta S \neq 0$. But the Hamilton function is obtained from the condition $\mathrm{d} \delta S=0$ which is a closure condition for the form $\delta S$. The transition from the Lagrange function $L$ to the Hamilton function $H$ (a transition from variables $q_{j}, \dot{q}_{j}$ to variables $\left.q_{j}, p_{j}=\partial L / \partial \dot{q}_{j}\right)$ is a transition from the tangent space, where the form is unclosed, to cotangent space with closed form. One can see that this transition is degenerate one (It should be noted that in the invariant field theories there used only nondegenerate transformations, which preserve the differential).

[By the example of canonical relations it is possible to show that nondegenerate and degenerate transformations are connected.

The canonical relations in the invariant field theory correspond to nondegenerate transformations. 
At the same time, the canonical relations for Equation (6) are integrability conditions obtained under degenerate transformation (when determinant becomes zero). In this case the degenerate transformation is a transition from the tangent space $\left(q_{j}, \dot{q}_{j}\right)$ to the cotangent (characteristic) manifold $\left(q_{j}, p_{j}\right)$ and is accompanied by the origin of physical structure. This proceeds spontaneously in evolutionary process if any degrees of freedom be realized.

On the other hand, the nondegenerate transformation is a transition from one characteristic manifold $\left(q_{j}, p_{j}\right)$ to the other characteristic manifold $\left(Q_{j}, P_{j}\right)$, that is, a transition from one physical structure to another physical structure. The nondegenerate transformations such as tangent, gradient, contact, gauge, conformal mapping, and others are transformations that are a method of describing invariant physical fields].

The first-order partial differential equation has been analyzed, and the functional relation with the form of the first degree has been considered.

Similar functional properties have all differential equations describing physical processes. And, if the order of the differential equation be $k$, the functional relation with the $k$-degree form corresponds to this equation.

In a similar way the ordinary differential equation can be investigated. In this case the integrability of the equation is connected with the consistency between derivatives and initial data.

\section{Investigation of Integrability of the Set of Differential Mathematical Physics Equations, Double Solutions of the Equations of Mathematical Physics}

As it was already noted, in present paper there are studied the mathematical physics equations on which no additional condition (conditions of integrability) are imposed. The continuous media (hydrodynamical, cosmological and others), physical and informational systems, consisted of numerous elements, are commonly described by a set of equations.

The integrability of the set of differential equations depends not only on consistency between derivatives, but also on consistency between equations of the set.

\subsection{Investigating the Consistency of Equations in the Set of Equations, Evolutionary Relation}

The hidden properties of the set of differential equations, which allows for describing discrete transitions, quantum jumps, emergence of various structures and so on, can be clarified by the example of equations for material media (continuous media) such as thermodynamical, gas-dynamical, cosmic and other ones [The material media (materia system) is a variety of elements that have internal structure and interact to one another. Examples of elements that constitute the material system are fluid particles, cosmic objects and others. As examples of material media it may be the thermodynamic, gas dynamical and cosmic systems, the systems of elementary particles and others]. 
It is known that the equations of mathematical physics for material media consist of the conservation law equations for energy, linear momentum, angular momentum, and mass [7] [8], that is, they are a set of equations.

It turns out that the equations of conservation laws for material media are not consistent.

As it is known, a material medium is characterized by physical quantities such as energy, pressure, density and others. But, since they relate to a single material medium, it has to exist a quantity that execute a connection between physical quantities. This quantity defines a state of given material medium. The state functionals like entropy, action functional, wave function and others are such functionals (Physical meaning of such functionals and the equations for functionals like entropy, action functional and the Pointing vector were considered in the paper [9] [10]).

[Entropy is such functional for thermodynamic and gas-dynamic media. But for thermodynamical medium this is an entropy depending on thermodynamic variables, whereas for gas-dynamic medium this is an entropy depending on space-time coordinates].

When studying the consistency of conservation laws, the conservation law equations are transformed into equations expressed in terms of state functionals.

One more peculiarity of present investigation is a using two frames of reference.

We introduce two frames of reference: the first is an inertial one (this frame of reference is not connected with the material medium), and the second is an accompanying one (this frame of reference is connected with the manifold built by the trajectories of the material medium elements) (The Euler and Lagrange frames of reference are examples of such frames).

Let us analyze the correlation of the equations that describe the conservation laws for energy and linear momentum.

In the inertial frame of reference the energy equation can be reduced to the form [7]:

$$
\frac{D \psi}{D t}=A_{1}
$$

where $D / D t$ is the total derivative with respect to time, $A_{1}$ is a quantity that depends on specific features of material medium and on energy actions onto the medium, $\psi$ is the state functional that specifies a material medium.

In the accompanying frame of reference the total derivative with respect to time is transformed into the derivative along the trajectory. Equation of energy is now written in the form:

$$
\frac{\partial \psi}{\partial \xi^{1}}=A_{1}
$$

Here $\xi^{1}$ is the coordinate along the trajectory.

In a similar manner, in the accompanying reference system the equation for linear momentum appears to be reduced to the equation of the form 


$$
\frac{\partial \psi}{\partial \xi^{v}}=A_{\nu}, v=2, \cdots
$$

where $\xi^{v}$ are the coordinates in the direction normal to the trajectory, $A$, are the quantities that depend on the specific features of material medium and on force actions.

Equations (9) and (10) can be convoluted into the relation

$$
\mathrm{d} \psi=\omega
$$

where $\omega=A_{\mu} \mathrm{d} \xi^{\mu}$ is the skew-symmetric differential form of the first degree (see Appendix 1).

In the general case (for energy, linear momentum, angular momentum and mass) this relation will be of the form:

$$
\mathrm{d} \psi=\omega^{p}
$$

where $\omega^{p}$ is the skew-symmetric form of degree $p$ that takes the values $p=0,1,2,3$.

The relations obtained and the skew-symmetric forms $\omega^{p}$ are evolutionary ones, since conservation law equations are evolutionary ones.

[A concrete forms of relations (11) and (12) and its properties were considered in the paper [11]. In the case of the Euler and Navier-Stokes equations the functional $\psi$ is the entropy $s$. A concrete form of relation (12) for $p=2$ was considered for electromagnetic field [12]. In this case the functional $\psi$ is the Pointing vector. The relation for Einstein's tensor is obtained when integrating the evolutionary relation for $p=3$ ].

\subsection{Nonidentity and Self-Changing of the Evolutionary Relation}

The evolutionary relation possesses nontraditional properties. This relation appears to be nonidentical and self-changing one.

Evolutionary relation proves to be nonidentical since the differential form in the right-hand side of this relation is not a closed form, and, hence, this form cannot be a differential like the left-hand side.

To justify this we shall analyze relation (11).

The skew-symmetric differential form, which is in the right-hand side, is not a closed form (its differential is not equal to zero) and cannot be a differential like the left-hand side. This relates to the fact that such skew-symmetric forms [3] (as opposed to exterior form which basis is an integrable manifold) is defined on accompanying manifold, which is a deforming non-integrable manifold. The differential of such a form contains a differential of the manifold metric form, which specifies a manifold deformation and hence is nonzero.

(For example, the differential $\mathrm{d} \omega$ of the form $\omega=A_{\mu} \mathrm{d} \xi^{\mu}$ can be represented as $\mathrm{d} \omega=K_{\alpha \beta} \mathrm{d} \xi^{\alpha} \mathrm{d} \xi^{\beta}$, where $K_{\alpha \beta}$ is the commutator of evolutionary form which can be written in the form (see Ref. [3] and Apendix 1):

$$
K_{\alpha \beta}=\left(\frac{\partial A_{\beta}}{\partial \xi^{\alpha}}-\frac{\partial A_{\alpha}}{\partial \xi^{\beta}}\right)+\left(\Gamma_{\beta \alpha}^{\sigma}-\Gamma_{\alpha \beta}^{\sigma}\right) A_{\sigma}
$$


Here $\Gamma_{\beta \alpha}^{\sigma}$ is a connectedness of accompanying manifold. The commutator $K_{\alpha \beta}$ is nonzero as the coefficients $A_{\mu}$ not potential, and connectedness of a accompanying manifold (which is a deforming non-integrable manifold) is notsymmetric [13]. Thus it turns out that the commutator of the form $\omega$ and its differentialis not equal to zero).

The non-identical evolutionary relation turns out to be self-changing one, that is, a change of one term leads to a change of another one and so on.

\subsection{Double Solutions of the Equations of Mathematical Physics}

The non-identity of evolutionary relation point to the fact that the conservation law equations appear to be inconsistent.

As it will be shown below, the nonidentity of evolutionary relation (caused by inconsistency of equations in the set of equations) as well as inconsistency of functional relation (2) (caused by inconsistency of derivatives of partial differential equation) points to non-integrability (without additional conditions) of the set of mathematical physics equations. This means that the corresponding solutions equations of mathematical physics have features.

Solutions to the mathematical physics equations on the original coordinate space (solutions that are not functions).

The nonidentical evolutionary relation $\mathrm{d} \psi=\omega^{p}$ cannot be integrated directly since its right-hand side contains unclosed skew-symmetric form which is not a differential. This means that the equations of mathematical physics prove to be non-integrable (they cannot be convoluted into identical relation for differentials and be integrated). In this case the solutions to equations of mathematical physics are not functions (their derivatives do not made up a differential). Such solutions will depend not only on variables. They will depend on the commutator of the evolutionary skew-symmetric form $\omega^{p}$ which is nonzero (If the commutator be equal to zero, the evolutionary relation would be identical and this would point out to integrability of original equations).

It turns out that solutions to the mathematical physics equations on the original coordinate space not a function. It depend on the commutator of the evolutionary skew-symmetric form $\omega^{p}$.

From the evolutionary relation it follows that the mathematical physics equations can become locally-integrable, i.e. they can have solutions that are functions.

Solutions to equations of mathematical physics on integrable structures (solutions that are discrete functions).

From the evolutionary relation it follows that the mathematical physics equations can have solutions that are functions. However, it is possible only in the case when from the evolutionary skew-symmetric form in the right-hand side of nonidentical evolutionary relation a closed skew-symmetric form, which is a differential, is realized. In this case the identical relation is obtained from the nonidentical relation, and this will point out to a consistency of the conservation law equations and an integrability of the mathematical physics equations. 
However, since the differential of evolutionary unclosed skew-symmetric form is nonzero, whereas the differential of closed exterior form is equal to zero, the closed exterior form can be obtained from evolutionary skew-symmetric form only under degenerate transformation, namely, under a transformation that does not conserve differential (The Legendre transformation is an example of such a transformation).

Degenerate transformations can take place under realization of any degrees of freedom. To this case it corresponds a conversion into zero of such functional expressions as determinants, Jacobians, the Poisson brackets, residues, and others. These conditions of degenerate transformation specify the integrable structures on which the solutions become discrete functions. The conditions of degenerate transformation can be realized under a change of nonidentical evolutionary relation, which, as it was noted, appears to be a self-varying relation.

If the conditions of degenerate transformation be realized, this means that it is realized an integrable structure on which from the unclosed evolutionary form $\omega^{p}$ (see evolutionary relation (12)) with non-vanishing differential $\mathrm{d} \omega^{p} \neq 0$, one can obtain a closed (inexact, only on integral structure) exterior form.

That is, it is realized the transition

$$
\mathrm{d} \omega^{p} \neq 0 \rightarrow(\text { degenerate transformation }) \rightarrow \mathrm{d}_{\pi} \omega^{p}=0, \mathrm{~d}_{\pi}^{*} \omega^{p}=0
$$

The realization of the conditions $\mathrm{d}_{\pi}{ }^{*} \omega^{p}=0$ and $\mathrm{d}_{\pi} \omega^{p}=0$ means that it is realized a closed dual form ${ }^{*} \omega^{p}$, which describes a certain integrable structure $\pi$ (which is a pseudostructure with respect to its metric properties), and the closed exterior (inexact) form $\omega_{\pi}^{p}$, which basis is a pseudostructure, is obtained.

On an integrable structure, from evolutionary relation (12) it follows the relation

$$
\mathrm{d} \psi_{\pi}=\omega_{\pi}^{p}
$$

which occurs to be an identical one, since the form $\omega_{\pi}^{p}$ is a differential.

Such relation can be integrated identically. This points out to the fact that the equations of mathematical physics become locally integrable (only on integrable structure).

The solutions to the mathematical physics equations on integrable structures are generalized solutions, which are discrete functions since they are realized only under additional conditions (on the integrable structures). The solutions on characteristics or on potential surfaces are examples of such generalized solutions.

On integrable structures the desired quantities of the material medium (such as the temperature, pressure, density) become functions of only independent variables and do not depend on the commutator (and on the path of integrating). Such functions may be found by means of integrating (on integrable structures) the equations of mathematical physics.

Since generalized solutions are defined only on realized integrable structures, they or their derivatives have discontinuities in the direction normal to integra- 
ble structure [6].

Thus, from the evolutionary relation it follows that the equations of mathematical physics have solutions of two types:

1) the solutions that are not functions, i.e. they depend not only on independent variables, and

2) the generalized solutions that are discrete functions.

The specific feature is the fact that these solutions are defined on different spatial objects. The solutions of the first type are defined on initial coordinate space, whereas generalized solutions are defined on integrable structures.

Such a peculiarity of the solutions to the equations of mathematical physics has a deep physical meaning, as it will be shown below, the double solutions describe the evolutionary processes proceeding in material medium, namely, the evolution of the material medium state that is accompanied by emergence of physical structures. and appearance of observable formations.

\section{Discrete Transitions, Emergence of Physical Structures and Observable Formations Such as Waves, Vortices, Turbulent Pulsations and Others}

In Section 2 it has been shown that double solutions to partial differential equations disclose a mechanism of discrete transitions, an emergence of quantum jumps and so on.

The double solutions to the mathematical physics equations for material media and physical systems that are composed of a few equations enable a description of the processes of discrete transitions and the emergence of physical structures and observable formations such as waves, vortices, turbulent pulsations and others.

\subsection{Emergence of Physical Structures}

It should be noted that closed dual forms and closed inexact exterior forms, which are realized under degenerate transformations, made up a differentialgeometric structure, i.e. a integrable structure (pseudostructure) with conservative quantity (closed exterior form describes a conservative quantity because its differential equals zero).

Such a differential-geometrical structure is an integrable structure, on which the solutions to the mathematical physics equations become functions. The structures like the characteristics, singular points, characteristic and potential surfaces, which are obtained when solving the mathematical physics equations, are such integrable structures.

On the other hand, such a differential-geometrical structure describes a pseudostructure with conservative quantity, i.e. a structure on which the exact conservation law [14] fulfills. Physical structures from which physical fields and relevant manifolds are formatted just are such structures [15] (Massless particles, structures made up by eikonal surfaces and wave fronts, and so on are examples 
of such physical structures).

The transition from the solutions on original coordinate space to the solutions on integrable structure and the realization of the differential-geometric structures describe a discrete transition and emergence of a certain physical structures. As already noted, this happens discretely, that is, by quanta.

\subsection{Description of the Material Medium State: An Advent of Observable Formations}

An unique importance of nonidentical evolutionary relation consists in the fact that this relation not only discloses a mathematical and physical meaning of double solutions to the equations of mathematical physics, but also describes of the evolutionary processes in material media, which are accompanied by the emergence of physical structures and the appearance of observed formations.

From the evolutionary relation it follows that this relation can describe the material medium state. This is due to the fact that in the left-hand side of the evolutionary relation there is the functional expression $\mathrm{d} \psi$ that determines the state of the material medium.

If the evolutionary relation proves to be identical, one can obtain the differential $\mathrm{d} \psi$ and find the state function $\psi$. This will indicate that the material medium state is in equilibrium. But since the evolutionary relation be nonidentical, this indicates an absence of the differential $d \psi$ and non-equilibrium of the material medium state [This can be shown by the example of Euler and Navier-Stokes equations that describe a flow of fluid and gases (i.e. a gas-dynamic medium) [11]. In this case a role of state functional play entropy. The evolutionary relation written in the form $\mathrm{d} s=\omega^{p}$ contains the differential of entropy ds. The presence of the differential of entropy means that in this case the entropy is a function of state, and this points out to equilibrium state of a gas-dynamic medium. However, from nonidentical relation one cannot obtain a differential of entropy. This means that entropy is not a state function. The absence of state function points out to non-equilibrium state of gas-dynamic medium].

The non-equilibrium means that an internal force acts in the material medium. It is evident that the internal force is described by the commutator of skew-symmetric form $\omega^{p}$ (If the evolutionary form commutator be zero, the evolutionary relation would be identical, and this would point to the equilibrium state, i.e. the absence of internal forces). Everything that gives a contribution into the evolutionary form commutator leads to appearance of the internal force.

A non-equilibrium state of material medium is described by the solution to the mathematical physics equations that are not functions.

Another property of the nonidentical evolutionary relation, namely, its selfvariation, points out to the fact that the non-equilibrium state of material medium turns out to be selfvarying (State of material medium changes but in this case remains to be non-equilibrium during this process, since the evolutionary relation remains to be nonidentical during the process of selfvariation). 
The realization of identical relation from evolutionary relation is indicative of the transition of material medium into the locally-equilibrium state. From identical relation one can find the differential of the state functional, and this points out to a presence of the state function and the transition of material medium from non-equilibrium state into equilibrium one. However, such a state of material medium turns out to be realized only locally due to the fact that differential of the state functional obtained is an differential interior (only on pseudostructure). A locally-equilibrium state of material medium is described by a generalized solution to the mathematical physics equations, i.e. a discrete function.

The transition from non-equilibrium state to locally equilibrium state means that unmeasurable quantity, which is described by the evolutionary form commutator and act as internal force, converts into a measurable quantity of material medium. This reveals in emergence of some observed formations in material medium, such as waves, vortices, fluctuations, turbulent pulsations and so on.

However, since proceeds of emergence of observable formations occur only locally, only a part of unobservable and immeasurable quantities converts into measurable quantities (observable formations) and a remainder part of unobservable and immeasurable quantities is kept in material medium (The dark energy and dark matter form such immeasurable and unobservable quantities [15]).

\subsection{Duality: Emergence of Physical Structures and Occurrence of Observed Formations}

As shown above, the transition from the solution on original coordinate space to the solution on integrable structure and the realization of the differential-geometric structure describe emergence of a certain physical structure. On the other hand, the transition from the solution on original coordinate space to the solution on integrable structure describe the transition of material medium state from nonequilibrium state into locally-equilibrium and emergence of some observable formations.

Thus, physical structures and observed formations are a manifestation of the same phenomenon (Light is an example of manifestation of such a duality, namely, as a massless particle (photon) and as a wave).

However, physical structures and observed formations are not identical objects. For example, when the wave arises as observable formation, the wave front is a physical structure (which is eikonal, i.e. a conserving surface). Thus whereas the wave is an observable formation, the element of wave front made up the physical structure in the process of its motion.

\section{Peculiarities of Numerical Modeling the Processes of Emergence of Various Structures}

To describe the process of emergence of various structures it is necessary have the solutions of the first type and the solutions of the second type (generalized solutions). 
The problems of numerical solving the mathematical physics equations relates to the fact that these solutions are defined on distinct spatial objects. For this reason they cannot be obtained by a continuous numerical simulation of derivatives.

The solutions of first type can be obtained only by numerical modeling the equations on the original non-integrable manifold (it is impossible to find such a solution by analytical method).

The solutions of the second type (generalized solution), cannot be obtained by modeling the equations on original manifold since they are obtained on integrable structures that do not belong to original non-integrable manifold.

To obtain the generalized solutions by numerical simulation, one must use the second frame of reference (on integrable structure). The generalized solutions can be obtained by analytical methods if the integrability conditions are imposed on the equations (The methods of characteristics, symmetries, eigen-functions and others are examples of such methods).

Therefore, to describe the emergence of various structures, quantum jumps and observable formations such as waves, vortices and so on, one must use two frames of reference or to apply two methods, namely, numerical and analytical ones.

One more problem of obtaining the generalized solution relates to the fact that the integrable structures with generalized solution are not initially given, and they are realized spontaneously in the process of integrating under the realization of additional conditions, namely, the integrability conditions (As additional conditions it may serve, for example, the characteristic conditions, the dynamical conditions of the consistency of equations in the set of equations and so on). To obtain the integrable structures, it is necessary to trace for the realization of additional conditions, which define the integrable structures, in the process of numerical integrating the equations on the original manifold.

The description of evolutionary processes is possible only either by numerical methods, but with two frames of reference, or by using simultaneously numerical and analytical methods [16].

\section{Conclusions}

It has been shown that the mathematical physics equations, on which no additional conditions (conditions of integrability) are imposed, can describe discrete transitions, quantum jumps, emergence of various structures and observable formations.

The conditions of integrability, which can be realized directly from the mathematical physics equations (if there are some degrees of freedom), are just connected with the properties of mathematical physics equations that allow a description of quantum processes.

These results were obtained when studying the integrability of mathematical physics equations that depend on the consistency of derivatives and the consistency of equations in the set of equations. 
In present paper it was shown that the description of quantum processes is connected with the following nontraditional peculiarities such as the realization of integrable structures, double solutions, and the transition from initial coordinate space to integrable structures.

It should be emphasized that such results were obtained with the help of skew-symmetric differential forms, which possess a nontraditional mathematical apparatus such as nonidentical relations, degenerate transformations and the transition from non-integrable manifolds to integrable structures.

Thus, it has been shown that the mathematical physics equations can describe not only a change of quatities of matherial media considered, physical systems and so on, but they also can describe an evolution of their state, namely, transitions from non-equilibrium state to locally-equilibrium one, that is accompanied by emergence of meashurable physical structures and observable formations. In the existing theory of mathematical physics equations there is no information about such properties of the mathematicaled physics equations. These results were obtained by the author with the help of skew-symmetric differential forms in which basis is non-integrable manifolds.

\section{Conflicts of Interest}

The author declares no conflicts of interest regarding the publication of this paper.

\section{References}

[1] Cartan, E. (1945) Les Systemes Differentiels Exterieurs et Leurs Applications Geometriques. Hermann \& Cie, Paris.

[2] Petrova, L.I. (2020) Skew-Symmetric Differential Forms. Conservation Laws. The Foundation of Equations of Mathematical Physics and Equations of Field Theory. KRASAND, Moscow.

[3] Petrova, L.I. (2010) Role of Skew-Symmetric Differential Forms in Mathematics. http://arxiv.org/pdf/1007.4757.pdf

[4] Bott, R. and Tu, L.W. (1982) Differential Forms in Algebraic Topology. Springer, New York. https://doi.org/10.1007/978-1-4757-3951-0

[5] Smirnov, V.I. (1957) A Course of Higher Mathematics. Vol. 4, Tech. Theor. Lit., Moscow. (In Russian)

[6] Petrova, L.I. (2009) Relationships between Discontinuities of Derivatives on Characteristics and Trajectories. Computational Mathematics and Modeling, 20, 367-372. https://doi.org/10.1007/s10598-009-9043-0

[7] Clarke, J.F. and Machesney, M. (1964) The Dynamics of Real Gases. Butterworths, London.

[8] Tolman, R.C. (1969) Relativity, Thermodynamics, and Cosmology. Clarendon Press, Oxford.

[9] Petrova, L.I. (2012) Physical Meaning and a Duality of Concepts of Wave Function, Action Functional, Entropy, the Pointing Vector, the Einstein Tensor. Journal of Mathematics Research, 4, 78-88. https://doi.org/10.5539/jmr.v4n3p78

[10] Petrova, L. (2018) Connection between Functions of the Field-Theory Equations 
and State Functionals of the Mathematical Physics Equations. Journal of Physics: Conference Series, 1051, Article No. 012025.

https://doi.org/10.1088/1742-6596/1051/1/012025

[11] Petrova, L.I. (2017) Features of Numerical Simulation of Euler and Navier-Stokes Equations. Computational Mathematics and Modeling, 28, 32-36.

https://doi.org/10.1007/s10598-016-9343-0

[12] Petrova, L.I. (2003) Exterior and Evolutionary Skew-Symmetric Differential Forms and Their Role in Mathematical Physics.

http://arxiv.org/pdf/math-ph/0310050v1.pdf

[13] Tonnelat, M.A. (1959) Les principles de la Theorie Electromagnetique et la Relativite. Masson, Paris.

[14] Petrova, L. (2013) Role of the Conservation Laws in Evolutionary Processes and Generation of Physical Structures. American Journal of Modern Physics, 2, 104-110. https://doi.org/10.11648/j.ajmp.20130203.12

[15] Petrova, L.I. (2020) Formatting Physical Fields and Pseudometric Manifolds. The Dark Matter. Journal of Physics: Conference Series, 1557, Article No. 012032.

https://doi.org/10.1088/1742-6596/1557/1/012032

[16] Petrova, L.I. (2012) Integrability and the Properties of Solutions to Euler and Navier-Stokes Equations. Journal of Mathematics Research, 4, 19-28.

https://doi.org/10.5539/jmr.v4n3p19 


\section{Appendix 1. Some Properties of Skew-Symmetric Forms Corresponding to the Conservation Laws}

Closed inexact exterior forms: differential-geometrical structures. The external differential form of the degree $p$ ( $p$-form) can be written as [4]:

$$
\theta^{p}=\sum_{i_{1} \cdots i_{p}} a_{i_{1} \cdots i_{p}} \mathrm{~d} x^{i_{1}} \wedge \mathrm{d} x^{i_{2}} \wedge \cdots \wedge \mathrm{d} x^{i_{p}}, \quad 0 \leq p \leq n
$$

Here $a_{i_{1} \cdots i_{p}}$ is the function of independent variables $x^{1}, \cdots, x^{n}, n$ is the space dimension, and $\mathrm{d} x^{i}, \mathrm{~d} x^{i} \wedge \mathrm{d} x^{j}, \mathrm{~d} x^{i} \wedge \mathrm{d} x^{j} \wedge \mathrm{d} x^{k}$, is the local basis subject to the condition of skew-symmetry:

$$
\begin{aligned}
& \mathrm{d} x^{i} \wedge \mathrm{d} x^{i}=0 \\
& \mathrm{~d} x^{i} \wedge \mathrm{d} x^{j}=-\mathrm{d} x^{j} \wedge \mathrm{d} x^{i}, \quad i \neq j
\end{aligned}
$$

The exterior form differential $\theta^{p}$ is expressed by the formula

$$
\mathrm{d} \theta^{p}=\sum_{i_{1} \cdots i_{p}} \mathrm{~d} a_{i_{1} \cdots i_{p}} \wedge \mathrm{d} x^{i_{1}} \wedge \mathrm{d} x^{i_{2}} \wedge \cdots \wedge \mathrm{d} x^{i_{p}}
$$

The form called as a closed one if its differential equals to zero:

$$
\mathrm{d} \theta^{p}=0
$$

From condition (A4) one can see that the closed form is a conservative quantity. This means that such a form can correspond to the conservation law (for physical fields), i.e. a conservative quantity.

If the form be closed only on pseudostructure, i.e. this form is a closed inexact one, the closure condition can be written as

$$
\mathrm{d}_{\pi} \theta^{p}=0
$$

In this case the pseudostructure $\pi$ obeys the condition

$$
\mathrm{d}_{\pi}^{*} \theta^{p}=0
$$

here ${ }^{*} \theta^{p}$ is the dual form.

From conditions (A5) and (A6) one can see that the dual form (pseudostructure) and closed inexact form (conservative quantity) describe a conservative object that can also correspond to some conservation law (It appears that the closed inexact exterior and dual forms describe a structure with conservative quantity. Such structures made up physical fields and pseudometric and metric manifolds).

It turns out that the closed inexact exterior forms are obtained from the skew-symmetric differential forms, whose basis is nonintegrable manifolds (in contrast to exterior skew-symmetric forms). Such forms, which possess the evolutionary properties, are obtained from the equations which describe any processes.

Distinction of evolutionary forms from exterior forms. The evolutionary form can be written in a manner similar for exterior differential form [2] [3]. However, in distinction from the exterior form differential, an additional term will appear in the evolutionary form differential. This is due to the fact that the evolutionary form basis changes since such a form is defined on nonintegrable 
manifold.

The evolutionary form differential takes the form

$$
\begin{aligned}
\mathrm{d} \theta^{p}= & \sum_{i_{1} \cdots i_{p}} \mathrm{~d} a_{i_{1} \cdots i_{p}} \wedge \mathrm{d} x^{i_{1}} \wedge \mathrm{d} x^{i_{2}} \wedge \cdots \wedge \mathrm{d} x^{i_{p}} \\
& +\sum_{i_{1} \cdots i_{p}} a_{i_{1} \cdots i_{p}} \mathrm{~d}\left(\mathrm{~d} x^{i_{1}} \wedge \mathrm{d} x^{i_{2}} \wedge \cdots \wedge \mathrm{d} x^{i_{p}}\right)
\end{aligned}
$$

where the second term is connected with the basis differential being nonzero: $\mathrm{d}\left(\mathrm{d} x^{i_{1}} \wedge \mathrm{d} x^{i_{2}} \wedge \cdots \wedge \mathrm{d} x^{i_{p}}\right) \neq 0$ (For the exterior form defined on integrable manifold one has $\left.\mathrm{d}\left(\mathrm{d} x^{i_{1}} \wedge \mathrm{d} x^{i_{2}} \wedge \cdots \wedge \mathrm{d} x^{i_{p}}\right)=0\right)$.

The peculiarity of skew-symmetric forms defined on nonintegrable manifold can be demonstrated by the example of a skew-symmetric form of first-degree. Let us consider the first-degree form $\omega=a_{\alpha} \mathrm{d} x^{\alpha}$. The differential of this form can be written as $\mathrm{d} \omega=K_{\alpha \beta} \mathrm{d} x^{\alpha} \mathrm{d} x^{\beta}$, where $K_{\alpha \beta}=a_{\beta ; \alpha}-a_{\alpha ; \beta}$ are components of the commutator of the form $\omega$, and $a_{\beta ; \alpha}, a_{\alpha ; \beta}$ are covariant derivatives. If we express the covariant derivatives in terms of connectedness (if it is possible), they can be written as $a_{\beta ; \alpha}=\partial a_{\beta} / \partial x^{\alpha}+\Gamma_{\beta \alpha}^{\sigma} a_{\sigma}$, where the first term results from differentiating the form coefficients, and the second term results from differentiating the basis. If we substitute the expressions for covariant derivatives into the formula for commutator components, we obtain the following expression for commutator components of the form $\omega$ :

$$
K_{\alpha \beta}=\left(\frac{\partial a_{\beta}}{\partial x^{\alpha}}-\frac{\partial a_{\alpha}}{\partial x^{\beta}}\right)+\left(\Gamma_{\beta \alpha}^{\sigma}-\Gamma_{\alpha \beta}^{\sigma}\right) a_{\sigma}
$$

Here the expressions $\left(\Gamma_{\beta \alpha}^{\sigma}-\Gamma_{\alpha \beta}^{\sigma}\right)$ entered into the second term are just components of the commutator of the first-degree metric form that specifies the manifold deformation and hence is nonzero [13] (It is well-known that the metric form commutators of the first-, second- and third-degrees specifies, respectively, torsion, rotation and curvature).

(In the commutator of exterior form, which is defined on integrable manifold, the second term absents: the connectednesses are symmetric, that is, the expression $\left(\Gamma_{\beta \alpha}^{\sigma}-\Gamma_{\alpha \beta}^{\sigma}\right)$ vanishes $)$.

Since the commutator, and hence the differential, of skew-symmetric form defined on nonintegrable manifold are nonzero, this means that such a form cannot be closed one.

The skew-symmetric form, which is obtained from the conservation law equations for material media, is just such evolutionary form. The basis of this form is an accompanying manifold, namely, the manifold built by the trajectories of the material system elements. Such a manifold is a deforming nonintegrable manifold. For this reason the differential of skew-symmetric form defined on such manifold is nonzero. Such evolutionary form is unclosed one. 